\title{
RESPECTFUL MATERNITY CARE (RMC): SAFE MOTHER HOOD
}

\author{
Hazarika $\mathbf{L}^{1}$ \\ ${ }^{1}$ Tutor, \\ Rahman Institute of Nursing and Paramedical \\ Sciences, \\ Guwahati, Assam, \\ India
}

Corresponding author: Lipika Hazarika

\author{
Konwar $\mathbf{G}^{2}$ \\ ${ }^{2}$ Professor, \\ Dean, Royal School of Nursing and Medical \\ and Allied sciences, \\ The Assam Royal Global University, \\ Guwahati, Assam, \\ India
}

Article DOI: https://doi.org/10.36713/epra6396

\begin{abstract}
Pregnancy and childbirth are the momentous events in the lives of every woman worldwide and represents a time of intense vulnerability. The concept of "Respectful Maternity Care (RMC)" has evolved and expanded over the past few decades to include diverse perspectives and frameworks. RMC is not only a crucial component of quality of care; it is a human right. A women's relationship with maternity care providers and the maternity care system during pregnancy and childbirth is vitally important. In 2014, WHO released a statement calling for the prevention and elimination of disrespect and abuse during childbirth, stating that "every woman has the right to the highest attainable standard of health, including the right to dignified, respectful care during pregnancy and childbirth." Traumatic childbirth experience can have many negative effects, including poor mother-baby bond, unwillingness to breastfeed and poor quality of life. As facility-based birth and the use of skilled birth attendants continue to rise, a focus on quality and RMC remains critical for improving global maternal health.
\end{abstract}

KEY WORDS: Safe Motherhood, maternity care, disrespect, abuse, childbirth

\section{INTRODUCTION}

Childbirth is the most important event in women's life and every woman need and deserve to receive respectful care during the labor and childbirth. ${ }^{1}$ The concept of "safe motherhood" is usually restricted to physical safety, and childbearing is also an important passage from woman to mother, with deep personal and cultural significance for a woman and her family. Because motherhood is specific to women, issues of gender equality and gender violence are also at the core of maternity care. ${ }^{2}$

There are several studies throughout the globe that attempted to explore the components of RMC during labor and birth from the laboring women's and providers' perspectives. These components generally include providing safe and timely care, nurturing positive interactions between midwives and women, protecting confidentiality, maintaining an active role in the labor process, obtaining the women's consent before performing procedures, providing information regarding procedures to women, respecting patient privacy, and promoting freedom of choice as it related to position for labor and birth. ${ }^{1}$

\section{UNIVERSAL CHILDBIRTH RIGHT}

1. Everyone has the right to freedom from harm and ill-treatment.

2. Everyone has the right to information, informed consent, and respect for their choices and preferences, including companion of choice during maternity care and refusal of medical procedures

3. Everyone has the right to privacy and confidentiality. No one is allowed to share

4. Everyone is their own person from the moment of birth and has the right to be treated with dignity and respect

5. Everyone has the right to equality, freedom from discrimination and equitable care. 
6. Everyone has the right to healthcare and to the highest attainable level of health.

7. Everyone has the right to liberty, autonomy, self-determination and freedom from arbitrary detention

8. Every child has the right to be with their parents or guardians

9. Every child has the right to an identity and nationality from birth.

10. Everyone has the right to adequate nutrition and clean water. ${ }^{3}$

\section{MIDWIVES PERSPECTIVES}

A qualitative study on Midwives' perspectives of respectful maternity care during childbirth was conducted in 2018 in two non-teaching public hospitals in Tehran, Iran. 24 semi-structured interviews were conducted with midwives, who had more than one year work experience in labor and childbirth units, through a purposive sampling method. Three themes were extracted including "showing empathy", "women-centered care" and "protecting rights". Showing empathy reflects that "establishing a friendly relationship" and "being with women". Women-centered care encompassed "keeping women safe" and "participating in decision making". Protecting rights reflected a need for "safeguarding dignity" as well as "giving equal care" and "preparing appropriate environment".

Iranian midwives considered respectful maternity care a broader concept than just preventing mistreatment. Providing supportive care through friendly interaction with women was the first step for providing respectful maternity care. ${ }^{1}$

\section{WOMEN PERSPECTIVES}

Child birth is a special moment for parents, families and communities but can also be a time of intense vulnerability for women to be exposed to disrespectful and abusive behavior while at the health facilities.

A cross sectional study on compassionate and respectful maternity care during facility based child birth was conducted in Bahir Dar, Ethiopia in 2017. Four public health centers and one referral hospital was randomly selected. A structured and pre-tested interviewer administered questionnaire was used to collect the data from 284 study participants using a systematic random sampling technique by allocating a proportion to each health facility. This study showed a high prevalence of disrespect and abuse during facility child birth in Bahir Dar town, Ethiopia. Being from rural area, having complications during delivery and mothers who gave birth through caesarian section were more likely to be exposed to disrespect and abuse than other women. ${ }^{2}$
Thus, the notion of safe motherhood must be expanded beyond the prevention of morbidity or mortality to encompass respect for women's basic human rights, including respect for women's autonomy, dignity, feelings, choices, and preferences, including companionship during maternity care. ${ }^{3}$

A systematic review and meta-analysis were conducted to identify various forms of ill-treatment, determinants, and pooled prevalence of disrespectful maternity care in India. After quality assessment, seven studies were included. Individual study prevalence ranged from $20.9 \%$ to $100 \%$. The overall pooled prevalence of disrespectful maternity care was $71.31 \%$. Pooled prevalence in community-based studies was $77.32 \%$ which was higher as compared to studies conducted in health facilities, this being $65.38 \%$. The highest reported form of ill-treatment was non-consent $(49.84 \%)$, verbal abuse $(25.75 \%)$ followed by threats $(23.25 \%)$, physical abuse $(16.96 \%)$, and discrimination (14.79\%). Besides, other factors identified included lack of dignity, delivery by unqualified personnel, lack of privacy, demand for informal payments, and lack of basic infrastructure, hygiene, and sanitation. The determinants identified for disrespect and abuse were sociocultural factors including age, socioeconomic status, caste, parity, women autonomy, empowerment, comorbidities, and environmental factors including infrastructural issues, overcrowding, ill-equipped health facilities, supply constraints, and healthcare access.

The high prevalence of disrespectful maternity care indicates an urgent need to improve maternity care in India by making it more respectful, dignified, and women-centered. Interventions, policies, and programs should be implemented that will protect the fundamental rights of women. ${ }^{4}$

A descriptive cross-sectional study was conducted among 150 mothers admitted to the maternity ward of Nepal Medical College and Teaching Hospital using a purposive sampling technique. Data were collected through an interview technique by using a validated tool containing 15 items each measured on a scale of 5 . In total, $84.7 \%$ of the women reported that they have experienced overall RMC services and, they also revealed that they have experienced disrespectful care in various forms such as being shouted upon (30.0\%), being slapped (18.7\%), delayed service provision (22.7\%), and not talking positively about pain and relief during childbirth (28.0\%). Likewise, length of stay, parity, and time of delivery were found as factors that influenced friendly care and timely care respectively. Even though RMC emphasizes eliminating disrespectful and abusive environment from health facilities, $15.0 \%$ of participants perceived that they have not experienced overall RMC services. So, the health facility should focus on the interventions which ensure that every woman receives this basic 
human dignity during one of the most vulnerable times in their lives. ${ }^{5}$

Disrespect and abuse (D\&A), a concept closely related to obstetric violence, has been documented in many different countries across the globe. In a 2010 landscape analysis, Bowser and Hill described 7 categories of disrespectful and abusive care during childbirth: physical abuse, non-consented clinical care, non-confidential care, non-dignified care, discrimination, abandonment and detention in health facilities. A 2015 systematic review updated this framework to include: Physical abuse, Sexual abuse, Verbal abuse, Stigma and discrimination, Failure to meet professional standards of care, Poor rapport between women and providers, Health system conditions and constraints.

Some evidence suggests that ethnic minorities are at greater risk of experiencing D\&A during facility-based childbirth. Other factors that might influence a woman's risk include socioeconomic status, parity, age and the partner's race. Being multiparous has been found to be a protective factor, which may suggest that past experience helps patients avoid disrespectful treatment, or that disrespectful treatment is normalized by past experiences among certain groups, such as women of color, young women, and those with economic, social or health challenges. ${ }^{6}$

Studies have reported significantly high prevalence of D\&A among women during pregnancy and childbirth in Nigeria, but little is known about women's perceptions and experiences of D\&A during maternity care in the country.

Five focus group discussions with a sample of 32 women were conducted as part of a qualitative phenomenological study. All the women received maternity care in health facilities in Benue State, Nigeria and had experienced at least one incident of disrespect and abuse. Audio-recorded discussions were transcribed and analyzed using a six-stage thematic analysis using NVivo11. The participants perceived incidents such as being shouted at and the use of abusive language as a common practice. Women described these incidents as devaluing and dehumanising to their sense of dignity. Some women perceived that professionals did not intend to cause harm by such behaviours. The women highlighted the importance of accessing health facilities for safe childbirth and expressed that the experiences of D\&A may not impact their intended use of health facilities. $^{7}$

\section{INFECTION CONTROL PRACTICES}

Postpartum infections result in maternal morbidities which includes a wide range of maternal complications such as septicemia, endotoxic shock, peritonitis or abscess formation leading to surgery and compromised future fertility. Sepsis accounts for approximately $10 \%$ of all maternal deaths. Puerperal sepsis causes at least 75000 maternal deaths every year, mostly in low-income countries. Infections during pregnancy are relatively prevalent, which are a result of alterations in pregnant women's cellmediated immunity. ${ }^{8}$

An Observational Study was being conducted to Understand Infection Risk during Childbirth in Kogi and Ebonyi States, Nigeria in six healthcare facilities. In each, five women were observed from the onset of labor through to delivery of the placenta. Hand hygiene infection risk was estimated for all procedures requiring aseptic technique compared against adherence to proper hand hygiene protocol and potential recontamination events. Hands were washed with soap and sterile gloves applied with no observed recontamination before only $3 \%$ of all observed procedures requiring aseptic technique. There was no significant difference in hygiene compliance between midwives and doctors nor facilities or states. Adherence to proper hygiene protocol was observed more in morning compared to afternoon and night shifts. This study highlights that hand hygiene remains a barrier to delivering high-quality and safe care in health facilities. Improving hygiene practices during labor and delivery will require strategies that extend beyond infrastructure provision. ${ }^{9}$

Another cross-sectional study was conducted on Infection control practices and program management in Labor and Delivery units in Zahedan, southeast Iran between March to September 2014 in teaching and non-teaching hospitals. A 108-item semi-structured questionnaire was used for data collection on 88 midwives. The evaluation scores for all aspects of infection control were suboptimal; infection control program management $38.1 \%$ to $71.4 \%$, healthcare workers post-exposure measures $58.5 \%$ to $92.7 \%$, medical waste management $73.9 \%$ to $87.0 \%$ and infection control related standard infrastructures and equipment's $55.9 \%$ to $82.8 \%$. The midwives mean scores for attending infection control training courses and hand hygiene were less than $40 \%$ of the maximum score, but the mean scores for normal vaginal deliveries scrub, and equipment's and instruments hygiene practices were generally above $70 \%$. Thus, the results of this study revealed a need for development of appropriate policies and protocols for infection control practices in labor and delivery units and also midwives training on clean delivery practices. $^{8}$

\section{COVID-19 PANDEMIC}

Respectful maternal care assumes great significance in times of a pandemic. Many cases have been reported by the media about pregnant women being denied admissions to hospitals, of being referred from one facility to another, with some even delivering by the roadside. 
Currently, women may shy away from accessing facilities due to fear of COVID-19 infections, and this may be compounded by fear of disrespect, discrimination and abuse, which is a more powerful deterrent to the use of skilled care than geographic and financial obstacles. It has taken years for India to get to the point where women from all socio-economic sections of society decide and opt for institutional deliveries instead of home births, and these gains must not be lost.

Women's experiences with maternity caregivers can empower and comfort them, or inflict lasting damage and emotional trauma. A woman's positive or negative memories of childbearing experiences stay with her throughout her lifetime. Studies shows that violating women's rights during childbirth leads women to distrust health care providers and facilities. Such women are not only less likely to seek out maternity care - such as postnatal and emergency obstetric care-but other health services as well, such as family planning. ${ }^{10}$

\section{EXPERIENCE SHARED BY PATIENTS}

Aruna, a young mother was forced to do the cleaner's job after her delivery: "I had just given birth and the pain was still too much to bear. They forced me to clean the floor of the labor room. I feel there is nothing worse than being a woman".

Contrary to common perception, even the wealthy and advantaged are sometimes not immune to being denied timely care and choice of a companion during labor and delivery, both essential components of respectful maternity care.

Television actress Chhavi Mittal has gone on record to say that she was denied a birth companion during her delivery, which, as per the guidelines of Government of India, should be ensured to a woman in labour. Other women have shared that “...When I could tolerate the pain, I asked for my mother, but I was refused and asked me to try on my own. I was not even allowed to hold my mom's hand". ${ }^{10}$

\section{NATIONAL NURSING AND MIDWIFERY COMMISSION BILL, 2020}

In December 2018, the Government of India took a landmark policy decision to launch the

Guidelines for Midwifery Services in India and to provide midwifery services through midwife-led care units (MLCUs) in all its LaQshya facilities by creating a specialist cadre of Nurse Practitioners in Midwifery (NPMs). The vision to have quality midwifery care for all mothers is grounded in the principles of respectful maternal care. These guidelines include the introduction of midwifery model of care for low-risk deliveries in midwiferyled units of public health facilities. The guidelines also lay emphasis on the requisite skills of NPMs in line with international standards and competencies. They also include guidelines for education and training of midwifery educators. In addition, they provide an understanding to integrate this model of care in the current public health system to contribute to achieving the SDGs.

SOMI, Society of Midwives, India makes the following suggestions for inclusion in the NNMC Bill 2020 and some are listed below-

1. Separate Midwifery Commission for Indian Midwifery or at least an exclusive Board for Midwifery Education and Practice constituted with eminent midwifery and public health leaders of the Country on par with international standards.

2. Initiating steps for launching of Direct Entry Midwifery Degree programme and Post Graduate programme with independent practice provisions.

3. Separate Cadre and career pathway and career progression from the position of Midwives to independent practitioners and Midwifery leaders.

4. Licensure for independent practice by NPMs who have been trained under different projects and pilot interventions to set up the first MLCUs as envisaged by Govt. of India.

5. Scope of Practice of Midwives to be clearly defined to run Midwife Led Care Units and Collaborative Midwifery Practice Units with adequate legal frame work.

6. Separate Register for Midwives at state and National level

7. To introduce nomenclature of community midwives for all ANMs with definite guidelines for upgrading them with training rather than creating a new category as Midwifery Associates.

8. Multi task responsibility of Nurses and midwives make dilution of skill. Therefore the skills and scope of practice of nurse and midwife should be clearly defined and sustained.

All nurses need not study midwifery, and midwives need not go through all the medical surgical and adult nursing knowledge and skills which they will never use in their practice. It is essential to clearly demarcate the education, practice scope and regulation of midwives as separate entities in the health system. ${ }^{11}$

\section{CONCLUSION}

Respectful maternity care (RMC) is a rightful expectation of every woman. On the contrary, problems such as disrespect, abuse, ill-treatment, and demand for informal payments, infrastructural issues such as lack of water supply, sanitation, electricity, 
and crowded rooms are prevalent globally. Another problem highlighted is that women either receive services "too much and too soon or too little and too late." Many women experience a lack of respectful and abusive care during childbirth across the globe.

Disrespectful care is therefore a topic of public health concern and has an effect on utilization of services, affects the progress of the country in terms of healthcare, and affects the mothers physically as well as psychologically. ${ }^{4}$

Health institutions face several challenges with an increased number of women delivering in a health facility. It necessitates a greater effort to upgrade the level of care provided to mothers along with their rights to noble and dutiful care. ${ }^{5}$

The White Ribbon Alliance, released the first charter on components of RMC including "respect for women's autonomy, dignity, empathy, privacy, confidentiality, feelings, choices, and preferences, including companionship during maternity care and continuous care during labor and childbirth and also prevention of harm and illtreatment." 4

RMC will help in achieving the Sustainable Development Goals, namely good health, well-being and reduced inequalities. ${ }^{4}$ Respectful care during childbirth has been described as "a universal human right that encompasses the principles of ethics and respect for women's feelings, dignity, choices, and preferences."

\section{REFERENCES}

1. Moridi $M$, Pazandeh $F$, Hajian S, Potrata B (2020) Midwives' perspectives of respectful maternity care during childbirth: A qualitative study. PLoS ONE 15(3): e0229941. https://doi.org/10.1371/journal.pone.0229941

2. Wassihun, B., Zeleke, S. Compassionate and respectful maternity care during facility based child birth and women's intent to use maternity service in Bahir Dar, Ethiopia. BMC Pregnancy Childbirth 18, 294 (2018). https://doi.org/10.1186/s12884-018-1909-8

3. https://www.whiteribbonalliance.org/wp content/uploads/2020/02/WRA_RMC_Brochure_ Desktop_8.5x11.pdf

4. Ansari $H$, Yeravdekar R. Respectful maternity care: A national landscape review. A national landscap Available from: http://www.nmji.in/text.asp?2019/32/5/290/2959 57

e review. Natl Med J India [serial online] 2019 [cited 2020 Nov 18];32:290-3.

5. Pratima Pathak, Bijaya Ghimire, "Perception of Women regarding Respectful Maternity Care during Facility-Based Childbirth", Obstetrics and Gynecology International, vol.2020, Article ID $\quad 5142398, \quad 8 \quad$ pages. https://doi.org/10.1155/2020/5142398

6. https://www.mhtf.org/topics/respectful-maternitycarel
7. Orpin, J., Puthussery, S., Davidson, R. et al. Women's experiences of disrespect and abuse in maternity care facilities in Benue State, Nigeria. BMC Pregnancy Childbirth 18, 213 (2018). $\quad$ https://doi.org/10.1186/s12884-0181847-5

8. Tabatabaei S M, Behmanesh Pour F, Azadeh $H$. Infection Control Practices and Program Management in Labor and Delivery Units: A Cross-Sectional Study From Iran, Int J Infect. 2016 ; 3(2):e32788. doi: 10.17795/iji-32788.

9. Buxton H, Flynn E, Oluyinka O, Cumming $O$, Esteves Mills J, Shiras T, Sara S, Dreibelbis R. Hygiene During Childbirth: An Observational Study to Understand Infection Risk in Healthcare Facilities in Kogi and Ebonyi States, Nigeria. Int $J$ Environ Res Public Health. 2019 Apr 11;16(7):1301. doi: 10.3390/ijerph16071301. PMID: 30979005; PMCID: PMC6479678.

10. https://poshan.outlookindia.com/story/poshannews-shame-on-us-for-treating-mothers-likethis/357819

11. National Nursing And Midwifery Commission Bill, 2020 , https://main.mohfw.gov.in/newshighlighs-21 